\title{
Effect of different contraction methods on pelvic floor muscle contraction in middle-aged women
}

\author{
Ji-Seon Kim ${ }^{a}$, Jong-Duk Choi ${ }^{b}$, Won-Seob Shin ${ }^{b}$ \\ ${ }^{a}$ Department of Physical Therapy, College of Health, Kyungwoon University, Gumi, Republic of Korea \\ ${ }^{b}$ Department of Physical Therapy, College of Health and Medical Science, Daejeon University, Daejeon, Republic of Korea
}

\begin{abstract}
Objective: Pelvic floor muscles (PFMs) form the base of the abdomino-pelvic cavity and also the PFMs function is important for urinary continence. PFMs training (PFMT) is considered to be the first method for PFM dysfunction. This study demonstrated correct PFMs contraction among commonly used different contraction methods for PFMT.

Design: Cross-sectional study.

Methods: In this study, nineteen middle-aged (40-70 years) women participated. To evaluate PFM function, ultrasonography was used to measure the distance of the bladder base movement. The distance of the PFM movements were calculated at rest and during the other contractions. The following four different contraction methods were performed randomly: (1) PFM contraction, (2) abdominal drawing-in maneuver (ADIM), (3) anal contraction, and (4) hip adductor muscle contraction. The participants held the contraction for 3 seconds for a total of 3 times with a 30 seconds rest period between each trial. The mean of three measurements in each position were obtained and compared with that in the resting position.
\end{abstract}

Results: The bladder base movement values were significantly greater when comparing PFM with ADIM and hip adductor contractions $(p<0.05)$. The bladder base movement values were significantly greater when comparing ADIM and anal contractions with hip adductor contractions $(p<0.05)$.

Conclusions: The results of this study suggest that performing PFM contractions is the best method among the common methods for PFMT. Performing PFM contractions was more effective than the other contraction methods.

Key Words: Pelvic floor, Ultrasonography, Urinary incontinence, Verbal reinforcement, Women

\section{Introduction}

Pelvic floor muscles (PFMs) form the base of the abdomino-pelvic cavity and provide mechanical support to the abdomino-pelvic viscera [1]. The important role of the PFMs is maintaining intra-abdominal pressure (IAP) and urinary continence during increased IAP [2,3]. Consequently, the PFMs function is important for urinary continence [2].

The treatments of urinary incontinence (UI) are surgery, medicine, magnetic treatment, and PFMs training (PFMT). Among these treatments, PFMT is considered as the first treatment of choice. Voluntary exercise for the PFMs was developed by Kegel [4], and then variations in the voluntary exercise program were used by physiotherapists. Various clinical training methods have been used in PFMT. The correct PFMs contraction must be an inward lift, not depression [5]. The most common training methods for PFMs are PFMs contraction, abdominal drawing-in maneuver (ADIM), anal contraction, and hip adductors muscle contraction [6]. Correct PFMs contraction should be performed in separation without abdominal or hip muscle activity [7]. Hip adductors and gluteus muscles contraction during the PFMT is considered incorrect [8]. However, no evidence has been found to support the effectiveness of these commonly used training methods facilitating correct PFMs contraction.

In advance research, ultrasonography has been used to as-

Received: 2 November, 2015 Revised: 26 November, 2015 Accepted: 26 November, 2015

Corresponding author: Won-Seob Shin

Department of Physical Therapy, College of Health and Medical Science, Daejeon University, 62 Daehak-ro, Dong-gu, Daejeon 34520, Republic of Korea Tel: 82-42-280-2294 Fax: 82-42-280-2295 E-mail: shinws@dju.kr

(c) This is an Open-Access article distributed under the terms of the Creative Commons Attribution Non-Commercial License (http://creativecommons.org/licens es/by-nc/4.0) which permits unrestricted non-commercial use, distribution, and reproduction in any medium, provided the original work is properly cited.

Copyright $@ 2015$ Korean Academy of Physical Therapy Rehabilitation Science 
sess PFMs function. Transabdominal ultrasonography (TAUS) is a common ultrasonographic method. TAUS has been found to be safe, non-invasive, and cost effective. TAUS measurements are quick and easy to apply, and patients are comfortable and do not need to undress [5].

To our knowledge, no study has compared PFMs function between the different PFMT methods (PFMs contraction, ADIM, anal contraction, and hip adductors muscle contraction) by using TAUS. The purpose of this study was to demonstrate correct PFMs contraction among the commonly used contraction methods for PFMT by using TAUS.

\section{Methods}

\section{Subjects}

A cross sectional study design was used to compare PFMs function between difference positions. In this study, 19 middle-aged women participated. The participants were aged between 40 and 70 years. The inclusion criteria were as follows: women who previously had a vaginal birth but not in the last 12 months and women who are able to follow verbal and written instructions in the Korean language. The exclusion criteria were as follow: women with a history of spinal or abdominal surgery, women with urinary tract or vaginal infections, and women with a known neurological disease. All of the participants provided written consent to participate. This study was approved by the Institutional Review Board of Daejeon University of Korea. The charac-

Table 1. General characteristics of the subjects

$(\mathrm{N}=19)$

\begin{tabular}{lr}
\hline \multicolumn{1}{c}{ Variable } & Mean (SD) \\
\hline Age $(\mathrm{yr})$ & $59.05(4.01)$ \\
Weight $(\mathrm{kg})$ & $59.58(5.92)$ \\
Height $(\mathrm{cm})$ & $155.00(5.13)$ \\
Body mass index $\left(\mathrm{kg} / \mathrm{m}^{2}\right)$ & $24.82(2.40)$ \\
\hline
\end{tabular}

teristics of all the participants are shown in Table 1.

\section{Research tools and data collecting process}

For diagnostic imaging of PFMs, a $3.5 \mathrm{~Hz}$ curved linear array ultrasonographic transducer was used. According to the bladder filling protocol, the participants consumed $600-750 \mathrm{ml}$ of water in 1 hour before the measurement. The measurement was performed in a crook-lying supine position. The lumbar spine was neutrally positioned, and the hips and knees were flexed to $60^{\circ}$, with one pillow beneath the head. The ultrasonographic transducer was placed transversely across the midline of the abdomen, immediately superior to the symphysis-pubis region. The angle of the transducer was moved toward the postero-inferior direction of the bladder until it is approximately $60^{\circ}$ from the vertical [9-12]. The distance of the PFMs movement was calculated at rest and during the other contractions. First, the marker ' $\mathrm{X}$ ' was placed on the bladder base at rest. Then, the participants performed PFMs contraction during the other PFMs contractions. The image was captured at the highest maximum displacement and again marked with a ' $X$ ' [9] (Figure 1). The difference between the resting position and the other task position's at the end of the bladder base was measured by using the on-screen calipers [12]. Resting position was used as a baseline measure for calculating the difference in PFMs contraction. In this study, assessment was conducted based on the participants' performance in the following resting position and four different contraction methods: (1) PFMs contraction, (2) ADIM, (3) anal contraction, and (4) hip adductor muscle contraction. These four contraction methods were performed randomly. Voluntary PFMs contractions were drawn in and lifted [6,9]. Through the ADIM, the lower abdominal wall was quietly drawn in toward the direction of the spine [6]. Anal contraction was performed by lifting and squeezing the anus. The last position was hip adductor muscle contraction, where the participant held a

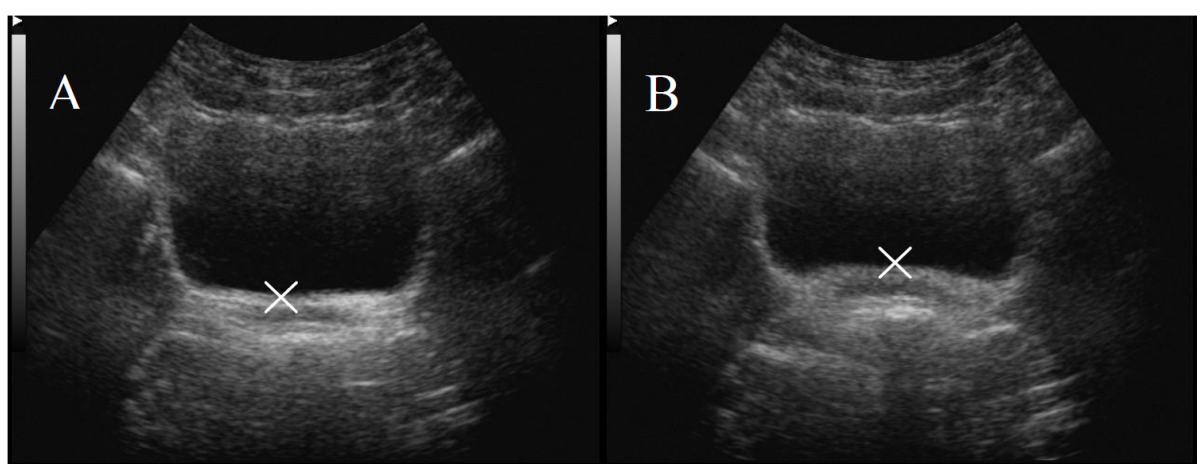

Figure 1. The transabdominal ultrasound images of bladder base. (A) Resting position. (B) Pelvic floor contraction. 
Table 2. Comparison of the bladder base's movement at the different verbal instruction

\begin{tabular}{cccccc}
\hline Variable & PFMC & ADIM & Anus & Hip adductor & F \\
\hline TAUS measurement $(\mathrm{cm})$ & $1.12(1.25)$ & $0.70(1.06)^{\mathrm{b}}$ & $1.04(1.57)$ & $0.25(0.82)^{\text {bcd }}$ & $10.627^{\mathrm{a}}$ \\
\hline
\end{tabular}

Values are presented as mean (SD).

PFMC: pelvic floor muscle contraction, ADIM: abdominal drawing-in maneuver, TAUS: transabdominal ultrasonography. ${ }^{\mathrm{a}} p<0.05$; ${ }^{\mathrm{b}}$ Significant difference in comparison with PFMC; ${ }^{\mathrm{c}}$ Significant difference in comparison with ADIM; ${ }^{\mathrm{d}}$ Significant difference in comparison with anus.

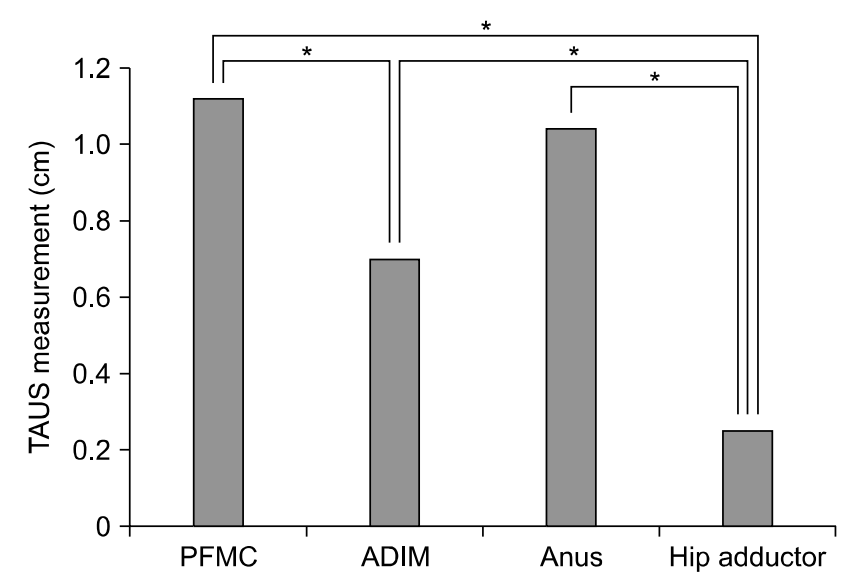

Figure 2. Characteristics of the subjects comparison of the bladder base's movement at the different contraction methods. TAUS: transabdominal ultrasonography, PFMC: pelvic floor muscle contraction, ADIM: abdominal drawing-in maneuver. ${ }^{*} p<0.05$.

ball between the sides of both knees in a crook-lying position. All contraction positions were performed while breathing normally [6]. The participants practiced each position prior to the data collection procedure. The mean of three measurements in each position that was measured by one investigator was used for the statistical analysis in this study. The participants held the contraction for no more than 3 seconds with 30 seconds rest time between each trial [9].

\section{Data and statistical analysis}

The collected data were statistically processed by using the PASW ver. 18.0 (IBM Co., Armonk, NY, USA). The general characteristics of the subjects were identified by using descriptive statistics. A repeated-measures analysis of variance was performed by using the mean ultrasonographic measurement for each method. The level of significance for all the analyses was set at $p<0.05$.

\section{Results}

Table 2 and Figure 2 present the bladder base movement values in the 4 different contraction tasks in difference with those in the resting position. In all the contraction tasks, the bladder base movement values increased from the values obtained at the resting position. Significant differences between the contraction tasks were found for movement values $(p<0.05)$, also significant interaction were observed in contraction tasks. Significant differences in the movement values were observed between PFMs contraction and ADIM, PFMs contraction and hip adductor contraction, ADIM and hip adductor contraction, then anal contraction and hip adductor contraction.

\section{Discussion}

Recently, PFMT has been used as the first treatment of choice for women with UI $[4,13]$. The aim of the PFMT is to improve the strength and endurance of the PFMs [14]. This study was designed to identify which among the commonly used contraction methods for PFMT by using TAUS induced good PFMs contraction.

Among the many different contraction methods used in PFMs contraction methods, the most commonly used contraction methods was chosen in this study. Except for hip adductor muscle contraction, the other contraction methods were more effective. These results are in agreement with those of the studies by Bø and Stien [15]. The gluteal and hip adductor muscles are important in daily activities, walking, and so on. In addition, these muscles facilitate the function of intrapelvic muscles such as PFMs [15]. ADIM is commonly used to activate deep abdominal muscles such as the transverses abdominus (TrA) [16]. PFMs is co-activated with deep abdominal muscles [17]. Urquhart et al. [18] found that PFMs contraction was activated $224 \%$ by the TrA. Similarly, Sapsford et al. [7] reported that the PFMs was activated during contraction of the abdominal muscles. Anal contraction is focused on anal muscle contraction [19]. The anal muscle consists of urethral support with PFMs. It maintains continence and pelvic support [20]. 
This study used TAUS for assessment of PFMs function. TAUS was used by physical therapists to assess PFMs function during movement of the bladder base that consequentially result in PFMs contraction $[1,5,21]$. TAUS has many advantages such as its safety, and non-invasiveness. In addition, this assessment technique is quick and easy to apply. These advantages are important in children, adolescents, men, and some ethnic groups, who may not benefit from assessment with digital palpation and transperineal ultrasonography into vaginal [9]. Thompson et al. [5] found a significant correlation between TAUS and transperineal ultrasonography, and manual muscle testing for PFMs contraction. Several studies have assessed PFMs function in women by using TAUS and transperineal ultrasonography $[12,22,23]$.

Our study has some limitations that should be improved in future studies. The small sample size may be a major limiting factor in generalizing the findings of this study. In addition, this study included healthy middle-aged women between 40 and 70 years old. The study results are difficult to generalize to the whole population with and without UI. The long-term effects of PFMT on the 4 different contraction tasks were not addressed because this study was completed during the initial effects. Therefore, future studies with larger study samples and longer intervention period are required to identify the clinical benefits of each method in the PFMT.

In conclusion, our study suggests that among the 4 different methods is the best method for facilitating PFMs contraction. Correct PFMs contraction is difficult to perform; therefore, correct contraction method to facilitate PFMs contraction is needed. The PFMs contraction method was more effective than the other commonly used contraction methods. This factor is suggested to increase the strength and endurance of the PFMs during rehabilitation. Further studies are required to develop management programs to facilitate correct PFMs contraction, and to investigate their effects.

\section{Conflict of Interest}

The authors declared no potential conflicts of interest with respect to the authorship and/or publication of this article.

\section{References}

1. Bø K, Sherburn M. Evaluation of female pelvic-floor muscle function and strength. Phys Ther 2005;85:269-82.

2. Smith MD, Coppieters MW, Hodges PW. Postural response of the pelvic floor and abdominal muscles in women with and without incontinence. Neurourol Urodyn 2007;26:377-85.

3. Sapsford RR, Hodges PW. Contraction of the pelvic floor muscles during abdominal maneuvers. Arch Phys Med Rehabil 2001;82:1081-8.

4. Kegel AH. Progressive resistance exercise in the functional restoration of the perineal muscles. Am J Obstet Gynecol 1948;56: 238-48.

5. Thompson JA, O'Sullivan PB, Briffa K, Neumann P, Court S. Assessment of pelvic floor movement using transabdominal and transperineal ultrasound. Int Urogynecol J Pelvic Floor Dysfunct 2005; 16:285-92.

6. Arab AM, Chehrehrazi M. Ultrasound measurement of abdominal muscles activity during abdominal hollowing and bracing in women with and without stress urinary incontinence. Man Ther 2011;16:596-601.

7. Sapsford RR, Hodges PW, Richardson CA, Cooper DH, Markwell SJ, Jull GA. Co-activation of the abdominal and pelvic floor muscles during voluntary exercises. Neurourol Urodyn 2001;20: 31-42.

8. Bump RC, Mattiasson A, Bø K, Brubaker LP, DeLancey JO, Klarskov $P$, et al. The standardization of terminology of female pelvic organ prolapse and pelvic floor dysfunction. Am J Obstet Gynecol 1996;175:10-7.

9. Arab AM, Behbahani RB, Lorestani L, Azari A. Assessment of pelvic floor muscle function in women with and without low back pain using transabdominal ultrasound. Man Ther 2010;15: 235-9.

10. Bø K, Sherburn M, Allen T. Transabdominal ultrasound measurement of pelvic floor muscle activity when activated directly or via a transversus abdominis muscle contraction. Neurourol Urodyn 2003;22:582-8.

11. Kelly M, Tan BK, Thompson J, Carroll S, Follington M, Arndt A, et al. Healthy adults can more easily elevate the pelvic floor in standing than in crook-lying: an experimental study. Aust $\mathbf{J}$ Physiother 2007;53:187-91.

12. Thompson JA, O’Sullivan PB, Briffa NK, Neumann P. Comparison of transperineal and transabdominal ultrasound in the assessment of voluntary pelvic floor muscle contractions and functional manoeuvres in continent and incontinent women. Int Urogynecol J Pelvic Floor Dysfunct 2007;18:779-86.

13. Roongsirisangrat S, Rangkla S, Manchana T, Tantisiriwat N. Rectal balloon training as an adjunctive method for pelvic floor muscle training in conservative management of stress urinary incontinence: a pilot study. J Med Assoc Thai 2012;95:1149-55.

14. Price N, Dawood R, Jackson SR. Pelvic floor exercise for urinary incontinence: a systematic literature review. Maturitas 2010;67: 309-15.

15. Bø K, Stien R. Needle EMG registration of striated urethral wall and pelvic floor muscle activity patterns during cough, Valsalva, abdominal, hip adductor, and gluteal muscle contractions in nulliparous healthy females. Neurourol Urodyn 1994;13:35-41.

16. Ferreira PH, Ferreira ML, Hodges PW. Changes in recruitment of the abdominal muscles in people with low back pain: ultrasound measurement of muscle activity. Spine (Phila Pa 1976) 2004;29:2560-6. 
17. Madill SJ, McLean L. Relationship between abdominal and pelvic floor muscle activation and intravaginal pressure during pelvic floor muscle contractions in healthy continent women. Neurourol Urodyn 2006;25:722-30.

18. Urquhart DM, Hodges PW, Allen TJ, Story IH. Abdominal muscle recruitment during a range of voluntary exercises. Man Ther 2005; 10:144-53.

19. Stafford RE, Ashton-Miller JA, Constantinou C, Coughlin G, Lutton NJ, Hodges PW. Pattern of activation of pelvic floor muscles in men differs with verbal instructions. Neurourol Urodyn 2015. doi: 10.1002/nau.22745. [Epub ahead of print]

20. Delancey JO, Ashton-Miller JA. Pathophysiology of adult urinary incontinence. Gastroenterology 2004;126:S23-32.
21. Sherburn M, Murphy CA, Carroll S, Allen TJ, Galea MP. Investigation of transabdominal real-time ultrasound to visualise the muscles of the pelvic floor. Aust J Physiother 2005;51:16770 .

22. Devreese A, Staes F, De Weerdt W, Feys H, Van Assche A, Penninckx F, et al. Clinical evaluation of pelvic floor muscle function in continent and incontinent women. Neurourol Urodyn 2004;23:190-7.

23. Thompson JA, O'Sullivan PB, Briffa NK, Neumann P. Assessment of voluntary pelvic floor muscle contraction in continent and incontinent women using transperineal ultrasound, manual muscle testing and vaginal squeeze pressure measurements. Int Urogynecol J Pelvic Floor Dysfunct 2006;17:624-30. 